called 'The impact of moral values on the promotion of science'. It was withdrawn because it substantially duplicated a 1999 paper $^{20}$ by Douglas Allchin, a historian and philosopher now at the University of Minnesota in Minneapolis.

Zohoor says that he never saw Allchin's paper, and that he only prepared a draft of the paper, leaving others in his office to "develop it and add the literature review". Zohoor says that the explanation of the staff member involved that the copying happened "quite accidentally and as a mere negligence" - is inadequate, and that he intends to write to Allchin to apologize. "In my entire life I've never copied anyone else's work," says Zohoor.

Declan Butler
Butler, D. Nature doi:10.1038/news.2009.945 (2009).

Butler, D. Nature 461, 578-579 (2009).

3. Ziari, H., Behbahani, H. \& Khabiri, M. M. Transport XXI, 207-212 (2006).

4. Ziari, H. \& Khabiri, M. M. J. Eng. Appl. Sci. 2, 33-37 (2007).

5. Kamal, M. A. Shazib, F. \& Yasin, B. J. East. Asia Soc. Transport. Stud. 6, 1329-1343 (2005).

6. Behbahani, H., Ziari, H. \& Noubakhat, S. J. Appl. Sci. Res. 4, 96-102 (2008).

7. Behbahani, H., Ziari, H. \& Noubakhat, S. J. Appl. Sci. Res. 4, 282-286 (2008).

8. Awwad, M. T. \& Shbeeb, L. Am. J. Appl. Sci. 4, 390-396 (2007).

9. Lucena, M. C. C., Soares, S. A. \& Soares, J. B. Mater. Res. 7, 529-534 (2004)

10.Emery, S. J. \& O'Connell, J. in Proc. 7th Conf. Asphalt Pavements for Southern Africa 29 August-2 September 1999, Victoria Falls, Zimbabwe (CAPSA, 1999).

11. Hofsink, W., Kong Kam Wa, N. Y. \& Dickinson, M. N. in Proc 8th Conf. on Asphalt Pavements for Southern Africa 12-16 September 2004, Sun City, South Africa (CAPSA, 2004).
COPENHAGEN LIVE

Nature's reporters blog from the United Nations climate conference.

go.nature.com/OEsfAa

\title{
Exoplanet claim bites the dust
}

Strike one planet from the list of 400-odd found around stars in other solar systems: a proposed planet near a star some 6 parsecs from Earth may not exist after all.

The finding is also a strike against a planet-seeking strategy called astrometry, which measures the side-to-side motion of a star on the sky to see whether any unseen bodies might be orbiting it. Ground-based astrometry has been used for more than a century, but none of the extrasolar planets it has detected has been verified in subsequent studies.

In May, Steven Pravdo of the Jet Propulsion Laboratory in Pasadena, California, and his colleagues raised fresh hopes for the technique when they announced an exoplanet, six times more massive than Jupiter, orbiting VB10, a star about one-thirteenth the mass of the Sun, using a telescope at the Palomar Observatory in southern California (S. Pravdo and S. Shaklan Astrophys. J. $700,623-632 ; 2009)$. But now a group led by Jacob Bean at the Georg-August University in Gottingen, Germany, has used a different approach, and found nothing. "The planet is not there," says Bean.

Bean and his colleagues used a well-honed technique called radial velocity, which has found most of the extrasolar planets detected so far. The method looks for shifts in the lines of a star's absorption spectrum to track its motion towards and away from Earth, which would be caused by the influence of a planet.

Radial-velocity measurements typically exploit the visible bands of the electromagnetic spectrum. But VB10 is a very dim star and gives off most of its light as infrared radiation. At the Very
Large Telescope in Chile, Bean placed a gas cell filled with ammonia in the path of the starlight, enabling him to calibrate the instrument for the infrared.

"We would definitely have seen a significant amount of variation in our data if [the planet] was there," says Bean, who has submitted the work to the Astrophysical Journal (J. L. Bean et al. Astrophys. J. preprint at http://fr.arxiv.org/abs/0912.0003; 2009).

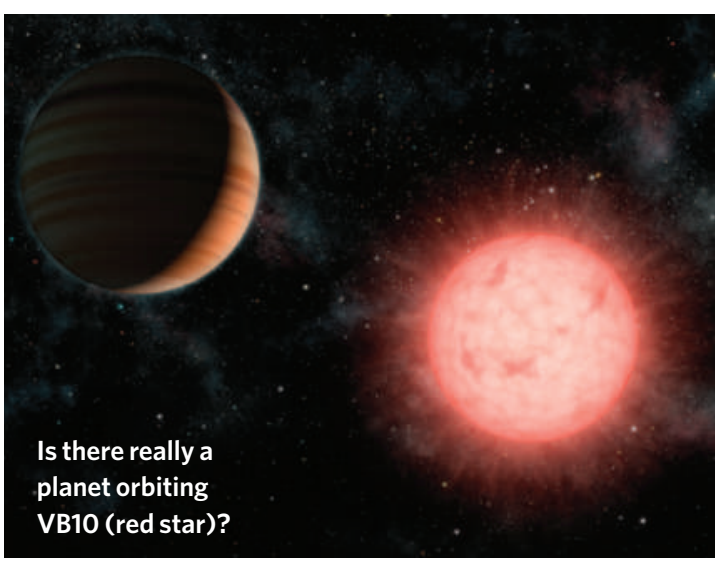

Pravdo says that Bean and his colleagues "may be correct, but there is hyperbole in their rejection of our candidate planet". Bean's paper, for instance, only rules out the presence of any planet that is at least three times more massive than Jupiter, says Pravdo, adding that the work "limits certain orbits for possible planets but not all planets".

"Unfortunately, astrometry is a very difficult business," counters Bean, explaining that Earth's atmosphere can introduce distortions that affect the measurements. Astrometrists rely on watching a field of stars about the same
12. Hanyu, A., Ueno, S., Kasahara, A. \& Saito, K. J. East. Asia Soc. Transport. Stud. 6, 1153-1167 (2005).

13. Fatehifar, E., Alizadeh Osalu, A., Kaynejad, M. A. \& Elkamel, A. in Proc. 3rd IASME/WSEAS Int. Conf. Energy \&Environment 23-25 February 2008, Univ. Cambridge, 330-335 (2008)

14. Lagzi, A. S. et al. in Air Pollution Modelling and Simulation (ed. Sportisse, B.) 264-273 (Springer, 2002).

15. Aliahmadi, A. R., Jafari, M. \& Amiri, B. in Proc. 2nd National Conf. Logistics \& Supply Chain 20-21 November 2006, Tehran (2006).

16. Hallikas, J., Karvonen, I., Pulkkinen, U., Virolainen, V.-M. \& Tuominen, M. Int. J. Prod. Econ. 90, 47-58 (2004).

17. Ojala, M. \& Hallikas, J. Int. J. Prod. Econ. 104, 201-213 (2006). 18. Harland, C., Brenchley, R. \& Walker, H. J. Purchasing Supply Management 9, 51-62 (2003)

19. The Experiences and Challenges of Science and Ethics: Proceedings of an American-Iranian Workshop (NAS, 2003). 20.Allchin, D. Sci. Educ. 8, 1-12 (1999).

See Editorial, page 699. distance away as the target star to calibrate their measurements, and that can be tricky, says Alessandro Sozzetti, an astrometry expert at the Turin Observatory in Italy. "Even if we think we have selected a good set of reference stars," he says, "we may still be limited by atmospheric effects that cause an extra jitter" in the motion of those stars.

Alan Boss, an exoplanet expert at the Carnegie Institution of Washington, agrees. He points to the well-known 'detection' of 1963, when Dutch astronomer Piet van de Kamp used astrometry to claim that two planets were orbiting Barnard's Star - a finding disproved a decade later. The dispute over the VB10 planet, says Boss, "is another example of how hard it is to detect extrasolar planets using astrometry from the ground".

Astronomers expect astrometry to work much better above the distorting effects of the atmosphere. Two space missions in the works - the European Space Agency's GAIA, due to launch in 2012, and NASA's Space Interferometry Mission, the launch date for which is yet to be set - will use the technique to search for planets as small as Earth around Sunlike stars, says Sozzetti. More significantly, astrometry can yield the mass of a planet, whereas radial velocity only puts a lower limit on it.

Bean admits that astronomers might one day find a planet around VB10 if they scrutinize the star long and hard enough. “The main lesson from VB10," says Boss, is that a lot of high-quality data are needed to be sure that an exoplanet is present. Katharine Sanderson 